\title{
Development of High Power All-Solid-State Red, Green and Blue Lasers
}

\author{
Dafu CUI, Jialin XU, Yong BO, Qianjin CUI, Qinjun PENG, and Zuyan XU \\ Laboratory of Optical Physics, Institute of Physics, Chinese Academy of Sciences, \\ Beijing 100080, China
}

(Received January 1, 2008)

\begin{abstract}
The high power all-solid-state red, green and blue lasers have been developed by means of Q-switched diode side-pumped Nd:YAG laser modules and the intracavity nonlinear frequency conversion in the LBO nonlinear crystals. The red laser generates based on the frequency doubled $1.3 \mu \mathrm{m} \mathrm{Nd:YAG} \mathrm{transition,} \mathrm{and} \mathrm{corresponding} \mathrm{maximum} \mathrm{output} \mathrm{power} \mathrm{is}$ $64 \mathrm{~W}$. The green output power of $218 \mathrm{~W}$ from frequency doubled $1064 \mathrm{~nm} \mathrm{Nd:YAG} \mathrm{transition}$ have been obtained. The blue laser based on the frequency tripled $1.3 \mu \mathrm{m} \mathrm{Nd:YAG} \mathrm{transition}$ delivers the output power of $7.6 \mathrm{~W}$. To best of our knowledge, this is the maximum blue output power for the intracavity frequency tripled $1319 \mathrm{~nm} \mathrm{Nd}$ :YAG laser.
\end{abstract}

Key Words: All-solid-state laser, High power laser, Red, Green, Blue.

\section{Introduction}

The high power compact all-solid-state red laser can be used as a source for laser therapy and pumping source for frequency conversion. ${ }^{1-3)}$ The green lasers with high power and high beam quality have in demand for precise material processing, bi-medicine and scientific application as pumping source for parametric oscillations or for fourth harmonic generation of solid-state lasers. ${ }^{4-6)}$ For all-solid-state blue laser sources, there exist numerous applications such as in high-density optical data storage, biological and medical diagnostics, underwater communication or underwater imaging. ${ }^{7)}$ Also, the compact high power all-state-solid red-green-blue (RGB) laser system is suitable for large image laser projection systems. ${ }^{8)}$

Here we report the high power quasi-continuous wave all-solid-state red, green and blue lasers. The red and green lights are generated by means of intracavity frequency doubled diode side-pumped $1064 \mathrm{~nm}$ and $1.3 \mu \mathrm{m} \mathrm{Nd:YAG} \mathrm{lasers} \mathrm{with} \mathrm{a}$ LBO nonlinear crystal, respectively. The blue laser is generated based on the diode side-pumped intracavity frequency tripled $1.3 \mu \mathrm{m}$ Nd:YAG laser with two LBO nonlinear crystals. Here, one LBO is for the second harmonic generation and other LBO crystal is for third harmonic generation. The maximum output power of the red laser is 64 $\mathrm{W}$ at $808 \mathrm{~nm}$ LD pump power of $650 \mathrm{~W}$. The green laser reaches up to the output power of $218 \mathrm{~W}$ at pump power of $930 \mathrm{~W}$ and the blue laser with the output power of $7.6 \mathrm{~W}$ have been obtained at the pump power of $480 \mathrm{~W}$.

\section{Experiment and results}

The high power all-solid-state quasi-continuous wave red, green and green lasers are generated by means of the acousto-optic Q-switched diode side-pumped Nd:YAG laser modules and intracavity nonlinear frequency conversion in the LBO nonlinear crystals. The acousto-optic Q-switch has an active aperture of $5 \mathrm{~mm}$, and their RF power is up to $100 \mathrm{~W}$. Pulsed operation is necessary for efficient of the intracavity frequency conversion. The high efficiency laser module consists of a Nd:YAG rod with $\mathrm{Nd}^{3+}$ doping concentration of 0.6 at. \% and five-fold symmetry diode arrays. Each array contains six $20 \mathrm{~W}$ continuous-wave diode bars. The diffusive reflector made of gold-filled copper is used for the homogeneous pumping. Figure 1 exhibits the schematic cross section of a diode-side-pumped laser module.

Figure 2 shows a typical fluorescence intensity distribution of a laser module measured with a CCD camera. Obviously, the fluorescence intensity distribution is uniform for our laser module. Effective and uniform pumping of the laser module is important for high average power and high beam quality laser generation. All of nonlinear crystals for second harmonic generation and third harmonic generation are the LBO because of its high optical damage threshold, low absorption at whole fundamental, second harmonic and third harmonic wavelength region, moderate nonlinear coefficients and sufficient birefringence to provide phase matching needed in our experiment. The LBO nonlinear crystal is placed in an oven, whose temperature is maintained by a precise temperature controller to a precision of $\pm 0.1 \mathrm{~K}$. The beam quality factor $\mathrm{M}^{2}$ is measured by a laser beam analyzer (Model $\mathrm{M}^{2}-200$, Spiricon, Inc.).

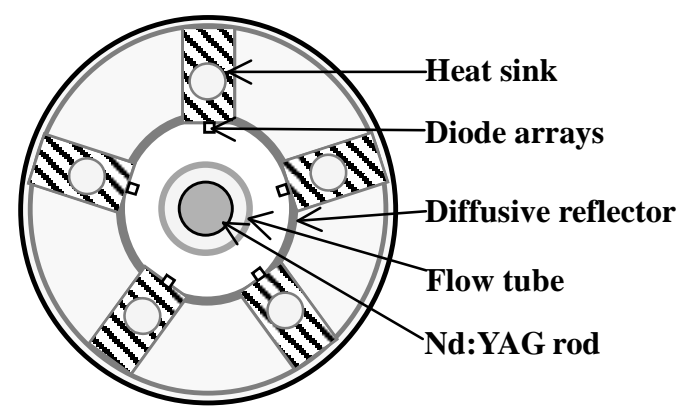

Fig.1 The schematic cross section of a diode-side-pumped $\mathrm{Nd}$ :YAG laser module. 


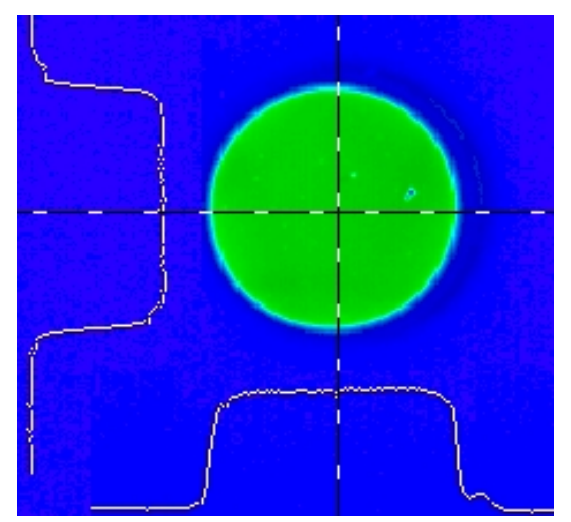

Fig.2 Typical fluorescence intensity distribution of the Nd:YAG laser module.

\subsection{Red laser}

The all-solid-state red laser is generated by using the intracavity frequency doubled $1.3 \mu \mathrm{m} \mathrm{Nd:YAG} \mathrm{laser.} \mathrm{A}$ L-shaped cavity laser resonator is composed of an acousto-optic Q-switch, two side-pumped Nd:YAG laser module, a quartz $90^{\circ}$ polarization rotator and a $4 \times 4 \times 40 \mathrm{~mm}^{3}$ LBO nonlinear crystal with type-II noncritical phase matching $\left(\theta=0^{\circ}, \Phi=0^{\circ}\right)$ at a temperature of $315.3 \mathrm{~K}$. The LBO crystal was coated dual-wavelength antireflection around $1.3 \mu \mathrm{m}$ and $660 \mathrm{~nm}$ simultaneously. The quartz $90^{\circ}$ polarization rotator is placed between two Nd:YAG rods of the laser modules for polarization dependent birefringence compensation.

The red output power as function of the $808 \mathrm{~nm}$ LD pump power is shown in Fig.3. The maximum average output power of the red laser is $64 \mathrm{~W}$ for the pump power of $650 \mathrm{~W}$ at repetition rate of $5 \mathrm{kHz}$ and pulse width of $161 \mathrm{~ns}$. We observe a roll over effect, i.e. output power up to the maximum and then falls with increasing pump power because of the Nd:YAG rods thermal effect.

As well known, $1.3 \mu \mathrm{m} \mathrm{Nd:YAG} \mathrm{laser} \mathrm{radiation} \mathrm{contains}$ two fundamental wavelengths. One is the $\mathrm{R}_{2} \rightarrow \mathrm{X}_{1}$ transition at the wavelength of $1.319 \mu \mathrm{m}$ and the other is the $\mathrm{R}_{2} \rightarrow \mathrm{X}_{3}$ transition at the wavelength of $1.338 \mu \mathrm{m}$. Their effective

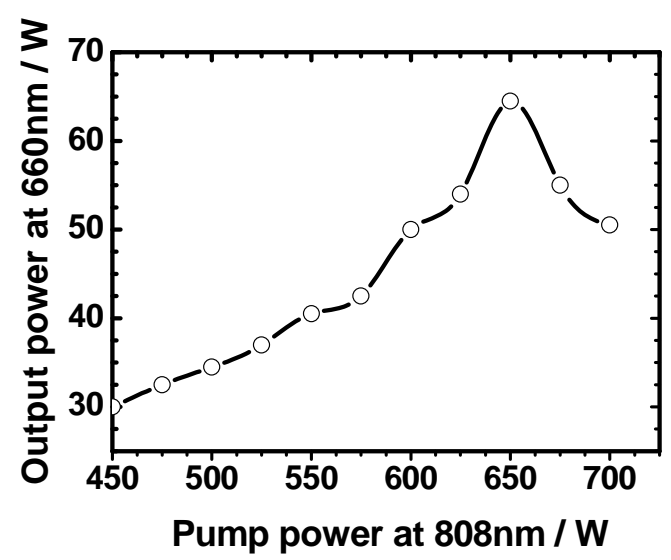

Fig.3 The red output power as function of the $808 \mathrm{~nm}$ LD pump power.

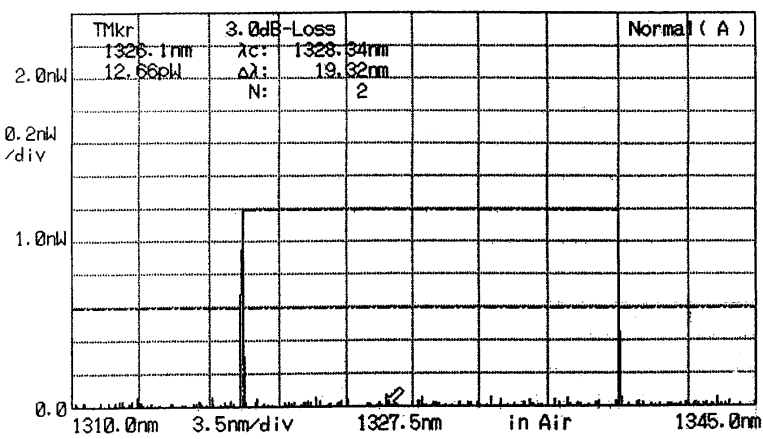

Fig.4 The single red spectrum at $659.5 \mathrm{~nm}$ is obtained at pump power of $500 \mathrm{~W}$.

stimulated emission cross section is nearly same, which is one third of the $R_{2} \rightarrow Y_{3}$ transition for the wavelength of $1.064 \mu \mathrm{m}$. Therefore, the red light obtained by using the frequency doubled $1.3 \mu \mathrm{m} \mathrm{Nd:YAG} \mathrm{laser} \mathrm{includes} \mathrm{both} 659.5 \mathrm{~nm}$ and $669 \mathrm{~nm}$ wavelengths while without wavelength selection element is employed.

Also, we have demonstrated the single wavelength 659.5 $\mathrm{nm}$ red light by using one side-pumped Nd:YAG laser module, and a etalon is inserted in the cavity in order to suppress the $\mathrm{Nd}$ :YAG laser $1.338 \mathrm{~nm}$ oscillation. The etalon was made of polished thin YAG without coating. YAG material, not silica, is selected because of its high refractive-index and perfect laser performance. It can improve the transmission difference between $1.319 \mu \mathrm{m}$ and $1.338 \mu \mathrm{m}$ from $12 \%$ to $28 \%$ than silica etalon. The insertion loss for the etalon at $1.319 \mu \mathrm{m}$ has been calculated to be less than $0.1 \%$.

We have measured the spectrum distribution of the red light by using Avantes mini fiber spectrum meter AvaSpec2048 with resolution power of $0.5 \mathrm{~nm}$. Under pump power of $500 \mathrm{~W}$, only single red spectrum at $659.5 \mathrm{~nm}$ can be seen as shown in Fig 4. The average output power of $28 \mathrm{~W}$ at $659.5 \mathrm{~nm}$ with beam quality factor of $\mathrm{M}^{2}=22$ is obtained for the pump power of $500 \mathrm{~W}$ at repetition rate of $5 \mathrm{kHz}$ and pulse width of 250 ns. ${ }^{9}$

\subsection{Green laser}

High power green laser can be achieved by external frequency doubling or intracavity frequency doubling a Q-switched Nd:YAG laser. In general, the former has better beam quality but its laser system is complicated. The later is simple and has higher conversion efficiency. In recent years, many more than $100-\mathrm{W}$ intracavity-frequency-doubled green lasers have been developed. Also, more than 200-W intracavity- frequency-doubled green laser obtained; however, the green beam exported out of two directions and the beam quality was not reported.

Here, a $218 \mathrm{~W}$ all-solid-state $532 \mathrm{~nm}$ green laser is obtained with Q-switched intracavity frequency doubled $1064 \mathrm{~nm}$ $\mathrm{Nd}$ :YAG laser based on a LBO nonlinear crystal. A thermally near-unstable resonator with two Nd:YAG rods in a L-shaped flat-flat cavity with a total length of $1050 \mathrm{~mm}$ is designed for the high output power and high beam quality. As same as the red laser system, a quartz $90^{\circ}$ polarization rotator is placed between two Nd:YAG rods of the laser modules for 


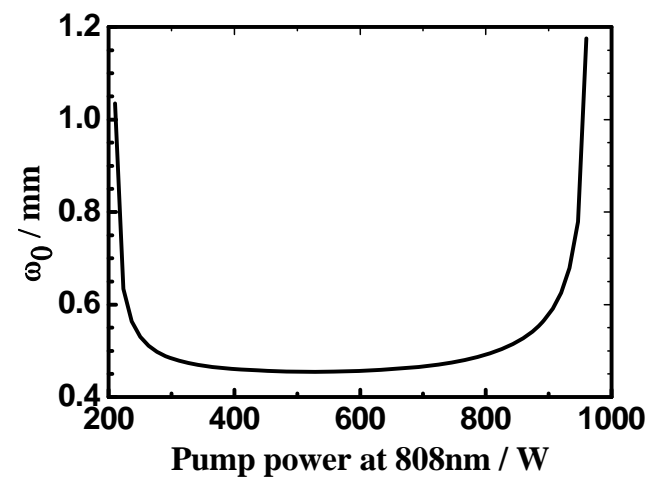

Fig.5 Calculated fundamental mode radius $\omega_{0}$ at the center of rods as the functions of the pump power.

polarization dependent birefringence compensation. Two orthogonal acousto-optic Q-switches are used to sustain fully holding off the laser gain in order to keep the pulse operation under high pomp power. A type II phase-matched $5 \times 5 \times 20$ $\mathrm{mm}^{3}$ LBO $\left(\theta=27.20^{\circ}, \Phi=90^{\circ}\right)$ a temperature of $373 \mathrm{~K}$ is employed for the second harmonic generation. The LBO crystal was coated dual-wavelength antireflection at $1064 \mathrm{~nm}$ and $532 \mathrm{~nm}$, simultaneously.

To achieve high power and high beam quality green laser, a thermally near-unstable resonator is used. In a thermally near-unstable resonator, the laser operates near the unstable region at the border of the thermally stable zones. The flat-flat cavity used in our experimental acts as a unstable resonator, which leads to more diffraction loss for high order transverse modes and the fundamental mode size at the gain medium is large. Then, both high beam quality and high average output power can be expected. ${ }^{10)}$

Figure 5 illustrates the dependence of the fundamental mode radius $\omega_{0}$ at the center of the rods on the pump power. The fundamental mode radius is calculated by using the standard $\mathrm{ABCD}$ ray propagation matrix as the function of the thermal lens focal length for the diode-pumped Nd:YAG rod. The dependence of the thermal lens focal length of the rod on the pump power is experimentally confirmed with the unstable-resonator method.

Figure 6 shows the dependence of $532 \mathrm{~nm}$ green output power versus the $808 \mathrm{~nm}$ diode pump power. The maximum average output power of the green laser reaches $218 \mathrm{~W}$ with pulse width of $86 \mathrm{~ns}$ and repetition rate of $12.5 \mathrm{kHz}$ under pump power of $930 \mathrm{~W}$, which operates just at the border of the thermally stable zones as seen in Fig.5. The measured green beam quality factor is $\mathrm{M}^{2}=6.2$ at output power of $120 \mathrm{~W}^{11)}$ and $\mathrm{M}^{2}=20.2$ at output power of $218 \mathrm{~W}$, respectively. To our knowledge, those beam quality is best results for hundred $\mathrm{W}$ level all-state-solid $532 \mathrm{~nm}$ green lasers with intracavity frequency doubling.

\subsection{Blue laser}

Power levels up to $2.8 \mathrm{~W}$ have previously been achieved for blue lasers based on frequency doubling of a neodymium laser, but are limited to lower power levels due to considerable reabsorption and thermal losses. Power levels up to $10.1 \mathrm{~W}$ have been reported by frequency doubling of tunable lasers in

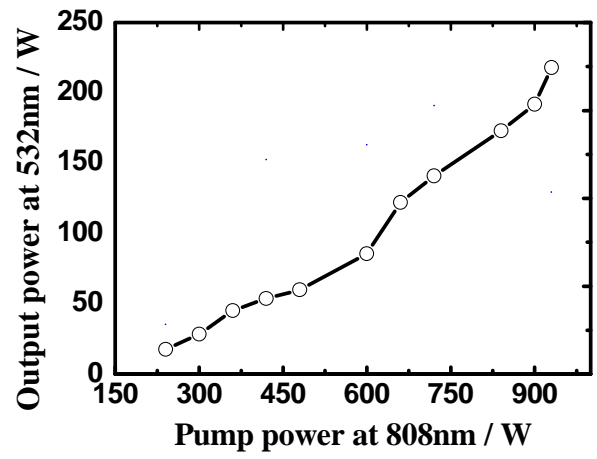

Fig.6 The $532 \mathrm{~nm}$ green output power as function of the LD pump power.

the near-IR region or by blue lasers produced directly through optical parametric oscillators (OPOs); however, these lasers are expensive and often too complicated for routine operation. Here, a simpler blue laser has been developed, that is based on frequency tripling of a compact Nd:YAG $1319 \mathrm{~nm}$ diode-pumped solid-state laser.

The all-solid-state blue laser is performed by using Q-switched intracavity frequency tripled $1319 \mathrm{~nm} \mathrm{Nd:YAG}$ laser. Figure 7 depicts the typical experiment configuration for the blue generation.

The laser resonator is composed of four mirrors, a acousto-optic Q-switch, two side-pumped Nd:YAG laser modules, a quartz $90^{\circ}$ rotator, a thin YAG etalon, a Brewster plane and two LBO nonlinear crystals. Here, one LBO crystal is for the second harmonic generation, which was cut into a 4 x $4 \times 20 \mathrm{~mm}^{3}$ with type I phase matching $\left(\theta=84^{\circ}, \Phi=0^{\circ}\right)$ at a temperature of $330 \mathrm{~K}$. Other LBO crystal is for third harmonic generation, which was cut into a $4 \times 4 \times 40 \mathrm{~mm}^{3}$ with type-II noncritical phase matching $\left(\theta=0^{\circ}, \Phi=0^{\circ}\right)$ at a temperature of 433.1 K. Both LBO crystals were coated three-wavelength antireflection around $1.3 \mu \mathrm{m}, 660 \mathrm{~nm}$ and $440 \mathrm{~nm}$, respectively.

In order to reach optimum second harmonic generation and

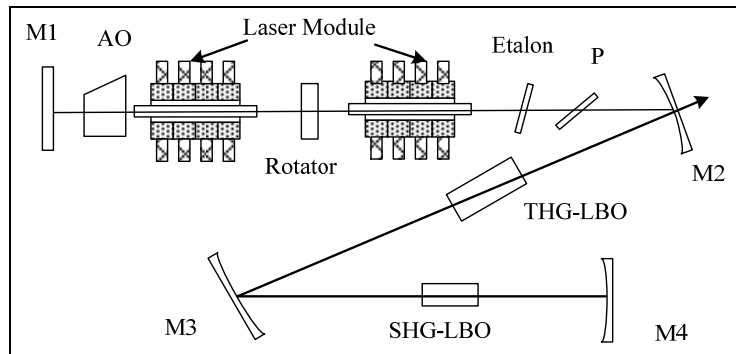

Fig.7 The schematic drawing of the experiment arrangement for the blue laser: $\mathrm{M} 1$ is the plane cavity mirror; M2,M3,M4, the concave mirrors; AO-QS, the acousto-optic Q-switch; Rotator, the quartz $90^{\circ}$ polarization rotator; $\mathrm{P}$, the Brewster plane; SHG-LBO, the nonlinear crystal for second harmonic generation; THG-LBO, the nonlinear crystal for third harmonic generation. 


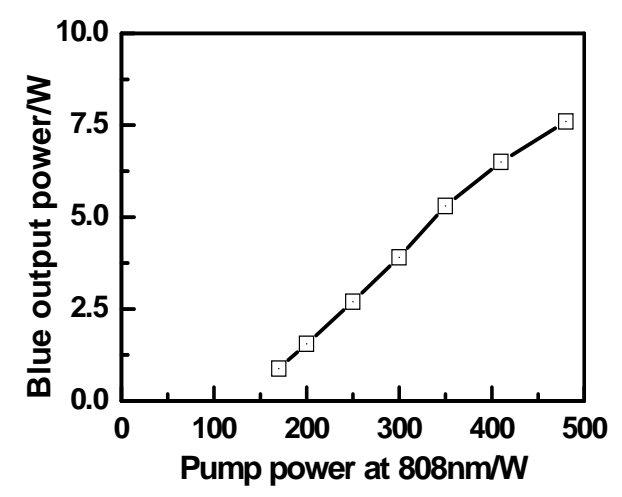

Fig.8.The $439.7 \mathrm{~nm}$ blue output power as function of the LD pump power.

third harmonic generation simultaneously, a special Z-type resonator, which has two focuses, was designed. Because $\mathrm{Nd}$ :YAG is an isotropic laser medium and emits unpolarized radiation, it is necessary to polarize the laser beam with a Brewster plane in order to meet the phase matching for maximize frequency conversion efficiency.

The LBO crystals are set close to those focuses, respectively. In the first LBO crystal (named as SHG-LBO in Fig. 7), some fraction of the fundamental radiation at $1319 \mathrm{~nm}$ is converted to the second harmonic radiation at $659.5 \mathrm{~nm}$. In another LBO crystal (named as THG-LBO in Fig.7), unconverted fundamental radiation is mixed with second harmonic to produce the third harmonic blue light at $439.7 \mathrm{~nm}$. The conversion efficiency and the stability of the blue laser output power are both improved through the suppression of $1338 \mathrm{~nm}$ $\mathrm{Nd}$ :YAG laser operation by means of the YAG etalon.

Experimentally, we measured the $439.7 \mathrm{~nm}$ blue output power by a laser meter (Ophir F300A). Figure 8 shows the blue output power as function of the $808 \mathrm{~nm}$ LD pump power. The blue output started at a pumping power around $170 \mathrm{~W}$. The blue laser delivers output power of $7.6 \mathrm{~W}$ at diode pump power of $480 \mathrm{~W}$ with the repetition rate of $5 \mathrm{kHz}$ and pulse width of $170 \mathrm{~ns}$. The slope efficiency is about $2.1 \%$.To best of our knowledge, this is the maximum blue output power for the intracavity frequency tripled $1319 \mathrm{~nm} \mathrm{Nd:YAG} \mathrm{laser.}$

We have measured the spectrum distribution of the blue light by using Avantes mini fiber spectrum meter AvaSpec 2048 with resolution power of $0.5 \mathrm{~nm}$. Under pump power of $480 \mathrm{~W}$, the spectrum only shows a single blue line at $439.7 \mathrm{~nm}$.

The far field intensity distribution of the $439.7 \mathrm{~nm}$ blue beam is exhibited in Fig.9, and the corresponding beam quality factor is measured to be $\mathrm{M}^{2}=12$ for both horizontal and vertical directions. At a lower output, the beam quality becomes better. For example, under an output power of $3 \mathrm{~W}$, the value of $\mathrm{M}^{2}$ is reduced to about 8 . The long-term power fluctuation is less than $1 \%$ at output power of $6 \mathrm{~W} .^{12)}$

\section{Conclusions}

The high power all-solid-state red and green lasers are developed by means of intracavity frequency doubled $1064 \mathrm{~nm}$ $\mathrm{Nd}$ :YAG laser and $1.3 \mu \mathrm{m} \mathrm{Nd:YAG} \mathrm{laser} \mathrm{in} \mathrm{the} \mathrm{LBO} \mathrm{nonlinear}$

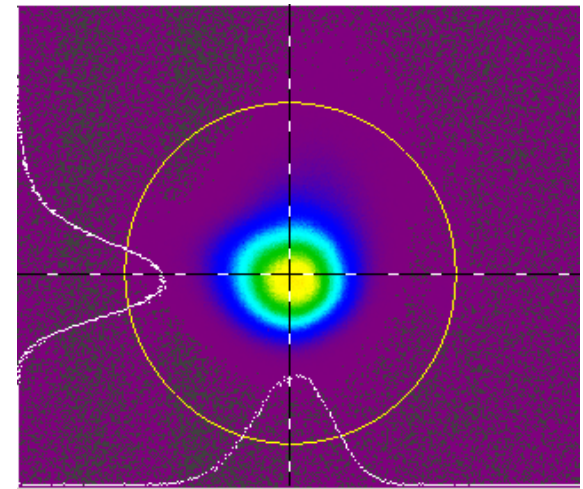

Fig.9 The far field intensity distribution of $439.7 \mathrm{~nm}$ blue beam.

crystals with type-II phase matching and type-II noncritical phase matching, respectively. The high power all-solid-state blue light is generated based on the intracavity frequency tripled $1.3 \mu \mathrm{m}$ Nd:YAG laser with two LBO nonlinear crystals, one LBO is for the second harmonic generation with type I phase matching and other LBO crystal is for third harmonic generation with type-II noncritical phase matching. The maximum average output power of the red laser reach $64 \mathrm{~W}$ at $808 \mathrm{~nm}$ diode pump power of $650 \mathrm{~W}$. The green laser output power is up to $218 \mathrm{~W}$ at pump power of $930 \mathrm{~W}$. The blue laser delivers the power of $7.6 \mathrm{~W}$ at pump power of $480 \mathrm{~W}$. To best of our knowledge, this is the maximum blue output power for the intracavity frequency tripled $1319 \mathrm{~nm} \mathrm{Nd:YAG} \mathrm{laser.}$

\section{Acknowledgment}

This work was supported by the State Key Program for Basic Research of China, the National High Technology Research and Development Program of China, the National Natural Science Foundation of China, and the Knowledge Innovation program of Chinese Academy of Sciences.

\section{References}

1) Z. Sun, R. Li, Y. Bi, X.Yang, Y. Bo, Y. Zhang, G. Wang, W. Zhao, H. Zhang, W. Hou, D. Cui, and Z. Xu; Opt.Commun. 241 (2004) 167.

2) A.Yao, W. Hou, X. Lin, Y. Bi, R. Li, D. Cui and Z. Xu; Opt.Commu. 231 (2004) 413

3) C. Du, S. Ruan, Y. Yu and F. Zeng, Opt. Express 13 (2005) 2013.

4) J. Golden: Laser Focus world 28 (1992) 75.

5) S. Konno, H.J. Moon and J. Lee: Opt. Lett. 25 (2000) 105.

6) H. Li, H. Zhang, Z. Bao, J. Zhang, Z. Sun, Y. Kong, Y. Bi, X. Lin, A. Y. Yao, G. Wang, W. Hou, R. Li, D. Cui and Z. Xu: Opt. Commun. 232 (2004) 411.

7) S. Bjurshagen, D. Evekull and R. Koch: Appl. Phys. B 76 (2003) 135.

8) Z. Xu and Y. Bi: Proc. SPIE. 5632 (2005) 115.

9) H. Peng, W. Hou, Y. Chen, D. Cui, Z. Xue, C. Chen, F. Fan and Y. Zhou: Opt. Express 14 (2006) 3961

10) Y. Feng, Y. Bi, Z. Xu, and G.. Zhang: Proc.SPIE 4969 (2003) 227.

11) Y. Bo, A. Geng, Y. Bi, Z. Sun, X. Yang, Q. Peng, H. Li, R. Li, D. Cui and Z. Xu: Appl. Opt. 45 (2006) 2499.

12) H. Peng, W. Hou, Y. Chen, D. Cui, Z. Xu, C. Chen, F. Fan and Y. Zhou: Opt. Express 14 (2006) 6543. 